\title{
Incidence and Risk Factors of Recurrence after Surgery for Pathology-proven Diverticular Disease
}

\author{
Caroline Andeweg · Joost Peters · Robert Bleichrodt • \\ Harry van Goor
}

Published online: 12 March 2008

(C) The Author(s) 2008

\begin{abstract}
Background Diverticular disease is a common problem in Western countries. Rationale for elective surgery is to prevent recurrent complicated diverticulitis and to reduce emergency procedures. Recurrent diverticulitis occurs in about $10 \%$ after resection. The pathogenesis for recurrence is not completely understood. We studied the incidence and risk factors for recurrence and the overall morbidity and mortality of surgical therapy for diverticular disease.

Methods Medical records of 183 consecutive patients with pathology-proven diverticulitis were eligible for evaluation. Mean duration of follow-up was 7.2 years. Number of preoperative episodes, emergency or elective surgeries, type of operation, level of anastomosis, postoperative complications, persistent postoperative pain, complications associated with colostomy reversal, and recurrent diverticulitis were noted. The Kaplan-Meier method was used to calculate the cumulative probability of recurrence. Cox regression was used to identify possible risk factors for recurrence.

Results The incidence of recurrence was $8.7 \%$, with an estimated risk of recurrence over a 15 -year period of $16 \%$. Risk factors associated with recurrence were (younger) age $(p<0.02)$ and the persistence of postoperative pain $(p<0.005)$. Persistent abdominal pain after surgery was present in $22 \%$. Eighty percent of patients who needed emergency surgery for acute diverticulitis had no
\end{abstract}

C. Andeweg $(\bowtie) \cdot$ R. Bleichrodt $\cdot$ H. van Goor Department of Surgery, Radboud University Medical Centre, Nijmegen, The Netherlands

e-mail: c.andeweg@chir.umcn.nl

J. Peters

Department of Surgery, Canisius Wilhemina Hospital, Nijmegen, The Netherlands manifestation of diverticular disease prior to surgery. In addition, recurrent diverticulitis was not associated with a higher percentage of emergency procedures.

Conclusion Estimated risk of recurrence is high and abdominal complaints after surgical therapy for diverticulitis are frequent. Younger age and persistence of postoperative symptoms predict recurrent diverticulitis after resection. The clinical implication of these findings needs further investigation. The results of this study support the careful selection of patients for surgery for diverticulitis.

\section{Introduction}

Diverticulosis is considered to be mainly a problem of old age, with a prevalence of $35-50 \%$ [1,2]. About $10-25 \%$ of patients with diverticulosis will develop diverticular disease in their lifetime [3, 4]. The clinical presentation of diverticular disease depends on the severity of the inflammatory process and whether complications are present. Complicated diverticulitis refers to the presence of perforation, obstruction, and abscess or fistula formation. Between 25 and $55 \%$ of the patients with complicated diverticulitis will require surgery during their initial hospitalization [5].

The current recommendation for patients with diverticular disease is elective surgery after the second documented episode of diverticulitis to prevent recurrent disease, because recurrence may lead to more complications and greater morbidity [5-7]. Recently, however, the necessity and timing of elective surgery has been debated with respect to recurrent disease and prevention of major complications [8, 9]. Elective surgery for diverticular 
disease has failed when there is a recurrence that adversely affects the patient's well-being. In the literature recurrence rates after appropriate resection of the sigmoid vary between 5 and $11 \%$ and a substantial number of these patients need urgent reoperation [10-14]. Identifying patients at risk for failure of resectional therapy would help to better select patients for elective surgery. Thus far, the level of anastomosis and age have been associated with recurrence, but data confirming this are scarce $[11,12]$.

The aim of this study was to assess the incidence and to identify possible risk factors for recurrence of diverticulitis in a large well-defined group of patients who underwent surgery for uncomplicated and complicated diverticulitis. Moreover, overall morbidity and mortality of surgical therapy for diverticular disease was evaluated.

\section{Patients and methods}

A consecutive series of patients who underwent surgery for diverticulitis in our department between 1985 and 2003 were identified from the Dutch pathology computer database using search terms "diverticular disease," "diverticulitis," and "diverticulosis." Medical records were reviewed and the following data were collected: number of preoperative episodes (number of episodes of diverticulitis requiring hospital admission before operation), emergency or elective surgery, type of operation [sigmoid resection, sigmoid resection with colostomy (Hartmann), left-sided hemicolectomy, anterior resection (AR), AR with colostomy, miscellaneous], level of anastomosis (colorectal or colosigmoidal), postoperative complications, complications associated with colostomy reversal, and recurrent diverticulitis. The policy of the department is to do a limited resection, restricted to the macroscopically diseased colon, determining the level of anastomosis.

Only major complications related to the surgical procedure and reoperations were noted. Anastomotic leak had to be confirmed by either radiographic enema, CT scan, or reoperation. Colostomies, time until reversal of the colostomy, and complications thereof were also recorded. Signs of active inflammation and the length of the resected specimen were noted from the pathology report. To complete follow-up, a questionnaire was sent to the patients' general practitioner (GP) and patients were interviewed by phone about recurrent diverticulitis, persistent complaints of left abdominal pain, and discomfort after initial surgery. Recurrent diverticulitis was defined as tenderness in the left lower abdomen, in combination with fever (temperature $\geq 38^{\circ} \mathrm{C}$ ), or, alternatively, a sedimentation rate, Creactive protein, or white blood cell count above normal values resulting into hospital admission. These findings had to be consistent with barium enema, colonoscopy, or CT findings. This study was conducted with the approval of the ethics board of our hospital and written informed consent was obtained from all patients in the study who received a questionnaire.

\section{Statistical analysis}

The $t$ test for two independent groups was used to test differences between patients with and without recurrence for statistical significance in case of quantitative variables. The $\chi^{2}$ test was used in case of qualitative variables, and the Fisher exact test was used in the case of $2 \times 2$ tables. To deal with the variable lengths of follow-up, the KaplanMeier product-limit method was used to calculate the cumulative time-related incidence of recurrent diverticulitis after resection. The endpoint was the recurrence of diverticulitis after resection. For those patients with no recurrence, the date was considered right-censored at the date of death or the end of the observational period. This method calculates incidence curves over time by using follow-up data from all individuals in the cohort, regardless of the duration of follow-up. A univariate Cox regression was used to study differences in the incidence curve for the following risk factors: age, gender, number of preoperative episodes, type of operation, emergency or elective surgery, level of anastomosis, length of resected specimen, and persistent complaints after surgery. The hazard ratios with 95\% confidence interval are presented. A value of $p$ less than 0.05 was considered statistically significant.

\section{Results}

\section{Demographics}

Two hundred twenty-two patients were identified from the computer database. Thirty-nine patients were excluded because of coexisting colonic malignancy or an alternative diagnosis (Crohn's disease or ulcerative colitis) in the definitive pathologic examination report. Follow-up was complete in the remaining 183 patients. Patients' demographics are listed in Table 1. Mean duration of follow-up was 7.2 years (range $=0-18$ years). Mean age at the time of operation was 63 years (range $=26-93$ years). Seventy-three patients (40\%) had emergency surgery including sigmoid resection with colostomy in 47 patients $(64 \%)$ and sigmoid resection with primary anastomosis in 26 patients $(36 \%)$. Patients underwent elective surgery $(60 \%)$ after completing diagnostic procedures, including barium enema ( $n=101)$ and/or coloscopy $(n=48)$ and/or CT scanning $(n=4)$. All patients undergoing elective surgery had previous complaints suggesting diverticular disease with 
Table 1 Patient demographics by recurrence of diverticulitis
$\mathrm{AR}=$ anterior resection

${ }^{*} p$ value by Fisher exact test

** $p$ value by $\chi^{2}$

*** $p$ value by $t$ test

a Adjusted for length of followup

${ }^{b}$ Recurrence occurred in 3 patients after reversal of the colostomy and in 1 patient with a colostomy who later underwent a subtotal colectomy because of multiple diverticula in the entire colon

\begin{tabular}{|c|c|c|c|c|c|c|c|}
\hline \multirow[t]{2}{*}{ Variable } & & \multirow[t]{2}{*}{$N$} & \multicolumn{2}{|c|}{ Recurrence } & \multicolumn{2}{|c|}{ No recurrence } & \multirow[t]{2}{*}{$\rho$-value ${ }^{\mathrm{a}}$} \\
\hline & & & $\mathrm{N}$ & $\%$ & $\mathrm{~N}$ & $\%$ & \\
\hline \multirow[t]{2}{*}{ Gender } & Female & 99 & 9 & 9 & 90 & 91 & \multirow[t]{2}{*}{$1.00^{*}$} \\
\hline & Male & 84 & 7 & 8.3 & 77 & 91.7 & \\
\hline \multirow[t]{2}{*}{ Operation } & Elective & 110 & 10 & 9 & 100 & 91 & \multirow[t]{2}{*}{$1.00^{*}$} \\
\hline & Emergency & 73 & 6 & 8.2 & 67 & 91.8 & \\
\hline \multirow{5}{*}{$\begin{array}{l}\text { Number of preoperative } \\
\text { episodes }\end{array}$} & 0 & 63 & 3 & 4.8 & 60 & 95.2 & \multirow[t]{5}{*}{$0.07^{* *}$} \\
\hline & 1 & 16 & 3 & 18.8 & 13 & 71.2 & \\
\hline & 2 & 88 & 7 & 8 & 81 & 82 & \\
\hline & 3 & 11 & 3 & 27 & 8 & 73 & \\
\hline & $>4$ & 5 & 0 & 0 & 5 & 100 & \\
\hline \multirow[t]{6}{*}{ Type of operation } & Sigmoid resection & 88 & 10 & 11.4 & 78 & 88.6 & \multirow[t]{6}{*}{$0.53^{* *}$} \\
\hline & Hartmann & 62 & $4^{\mathrm{b}}$ & 6.5 & 58 & 93.5 & \\
\hline & $\begin{array}{l}\text { AR and primary } \\
\text { anastomosis }\end{array}$ & 12 & 2 & 11.1 & 10 & 88.9 & \\
\hline & $\mathrm{AR}$ and colostomy & 6 & 0 & 0 & 6 & 100 & \\
\hline & Miscellaneous & 10 & 0 & 0 & 10 & 100 & \\
\hline & $\begin{array}{l}\text { Left-sided } \\
\text { hemicolectomy }\end{array}$ & 5 & 0 & 0 & 5 & 100 & \\
\hline \multirow{2}{*}{$\begin{array}{l}\text { Signs of active } \\
\text { inflammation at } \\
\text { pathology }\end{array}$} & Yes & 166 & 13 & 7.8 & 153 & 92.2 & \multirow[t]{2}{*}{$0.17^{*}$} \\
\hline & No & 17 & 3 & 17.6 & 14 & 82.4 & \\
\hline \multirow[t]{2}{*}{ Persistent complaints } & Yes & 36 & 8 & 22.2 & 28 & 77.8 & \multirow[t]{2}{*}{$<0.01^{*}$} \\
\hline & No & 147 & 8 & 5.4 & 139 & 94.6 & \\
\hline \multirow[t]{5}{*}{ Level of anastomosis } & Colorectal & 21 & 3 & 14.3 & 18 & 85.7 & \multirow[t]{4}{*}{$0.04^{* *}$} \\
\hline & Colosigmoidal & 90 & 12 & 13.3 & 78 & 86.7 & \\
\hline & Colostomy & 68 & 1 & 1.5 & 67 & 98.5 & \\
\hline & Other & 4 & 0 & 0 & 4 & 100 & \\
\hline & & \multicolumn{5}{|c|}{ Mean } & \\
\hline \multirow[t]{2}{*}{ Age } & Recurrence & \multicolumn{5}{|c|}{54 years $($ range $=33-75)$} & \multirow[t]{2}{*}{$<0.02^{* * *}$} \\
\hline & No recurrence & \multicolumn{5}{|c|}{64 years $($ range $=27-93)$} & \\
\hline \multirow{2}{*}{$\begin{array}{l}\text { Length of resected } \\
\text { specimen }\end{array}$} & Recurrence & \multicolumn{5}{|c|}{$19.6 \mathrm{~cm}($ range $=12-34)$} & \multirow[t]{2}{*}{$0.16^{* * * *}$} \\
\hline & No recurrence & \multicolumn{5}{|c|}{$17.1 \mathrm{~cm}($ range $=7-35)$} & \\
\hline
\end{tabular}

one to more than four documented episodes. The median documented number of episodes in the total group was 2 (range $=0-10)$. Signs of active inflammation were present in 166 patients $(91 \%)$ at pathology.

\section{Postoperative morbidity and mortality}

Early postoperative complications (within 30 days after primary surgery) requiring reoperation occurred in nine patients $(4.9 \%)$. Indications for reoperation were anastomotic leak ( $n=3)$, abscess formation with abdominal sepsis $(n=3)$, gauze left behind at initial laparotomy $(n=1)$, iatrogenic bowel perforation $(n=1)$, and fascial dehiscence $(n=1)$. The 30-day mortality rate for patients who had emergency surgery was $9.5 \%(n=7)$. No patients died within 30 days after elective surgery. Sixty-eight (37.2\%) patients had a colostomy, which was reversed in $36(53 \%)$ after a mean of 10 (range $=1-63$ ) months. One patient died after colostomy reversal due to cardiogenic shock. Mean age of the group with colostomy reversal was significantly lower than the group who did not have colostomy reversal (60 vs. 69 years; $t$ test; $p=0.01)$. Three patients $(8.3 \%)$ had anastomotic leak resulting in reoperation.

\section{Persistent abdominal pain}

Thirty-four patients died before the end of the observational period, leaving 149 patients available for long-term follow up. Of this group, 33 patients $(22.1 \%)$ complained of persistent abdominal pain after primary resection. Signs of active inflammation were present at pathology in 29 of them $(88 \%)$. One hundred sixteen patients did not have 
persistent postoperative pain but signs of inflammation were present in a similar number (103 patients, 89\%). The mean length of resected sigmoid in the group with persistent postoperative pain was $17 \mathrm{~cm}$ compared to $17.5 \mathrm{~cm}$ in patients without complaints. ( $t$ test; $p=0.15$ )

\section{Recurrences}

Recurrence rate was $8.7 \%(n=16)$ and recurrences occurred after a mean of 3.2 (range $=0.5-12$ ) years. Mean age at operation was significantly lower in the recurrence group than in the nonrecurrence group (54 vs. 64 years; $t$ test; $p<0.01$ ). The type of previous operation performed in the recurrence group was resection of the sigmoid in ten cases, Hartmann's procedure in four cases, and a low anterior resection in two cases. Six of 16 (37.5\%) were emergency procedures. Eight patients $(50 \%)$ with recurrent disease were treated conservatively because the complaints were mild in seven patients and the remaining patient was in poor general condition not favoring surgery. The other eight patients underwent a left-sided hemicolectomy because of multiple diverticula in three, a partial resection of the transverse colon after inflammation induced stenosis in two, and active inflammation in one patient. It was necessary to conduct a subtotal colectomy in two cases because of multiple diverticula in the entire colon. Only two patients $(12.5 \%)$ with recurrent diverticulitis were operated on in an emergency setting.

Figure 1 shows the Kaplan-Meier estimator of the cumulative time-related incidence of recurrence. The estimated risk of recurrent diverticulitis 1 year after operation was $3 \%$ (standard error $[\mathrm{SE}]=1.3$ ), increasing to $8.2 \%$ $(\mathrm{SE}=2.3)$ at 5 years, $12 \%(\mathrm{SE}=3.0)$ at 10 years, and $16 \%(\mathrm{SE}=3.7)$ at 15 years

Table 2 shows the hazard ratios (with $95 \%$ confidence interval) of the risk factors for recurrent diverticulitis

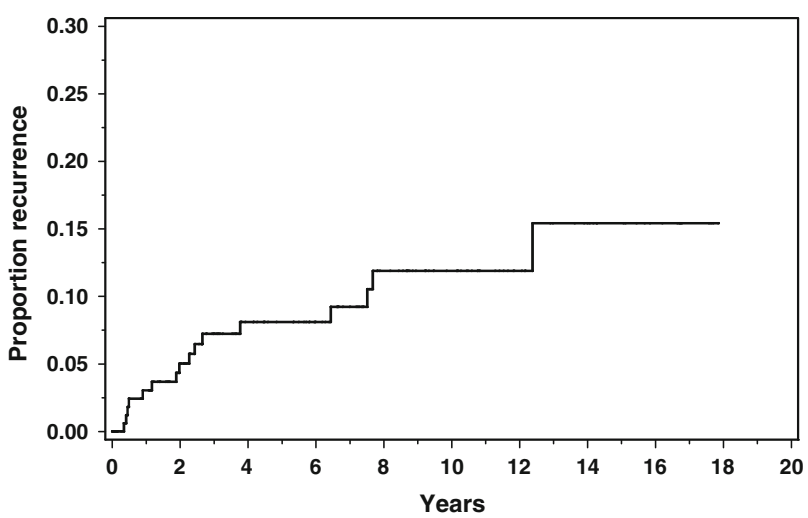

Fig. 1 Cumulative incidence of recurrent diverticulitis after resection using the Kaplan-Meier method for time-related incidence. This method adjusts the incidence ratio to account for various lengths of follow-up and losses to follow-up
Table 2 The hazard ratio with $95 \%$ confidence interval for recurrence of diverticulitis

\begin{tabular}{|c|c|c|}
\hline & Hazard ratio $(95 \% \mathrm{CI})$ & $p$ \\
\hline Gender & & 0.86 \\
\hline Female & $1.09(0.41-2.94)$ & \\
\hline Male & 1.00 (reference) & \\
\hline Operation & & 0.60 \\
\hline Elective & 1.00 (reference) & \\
\hline Emergency & $1.31(0.47-3.61)$ & \\
\hline Episodes (number) & $1.20(0.71-2.4)$ & 0.49 \\
\hline Type operation & & 0.34 \\
\hline Sigmoid resection & 1.00 (reference) & \\
\hline Hartmann & $0.72(0.23-2.30)$ & \\
\hline Anterior resection $^{\mathrm{a}}$ & $1.33(0.29-6.09)$ & \\
\hline Miscellaneous $^{\mathrm{b}}$ & $\mathrm{NE}$ & \\
\hline \multicolumn{2}{|c|}{ Signs of active inflammation at pathology } & 0.30 \\
\hline Yes & $0.48(0.14-1.69)$ & \\
\hline No & 1.00 (reference) & \\
\hline Persistent complaints & & $<0.01$ \\
\hline Yes & $4.76(1.79-12.5)$ & \\
\hline No & 1.00 (reference) & \\
\hline Level of anastomosis & & 0.02 \\
\hline Colorectal & $11.35(1.18-109.50)$ & \\
\hline Colosigmoidal & $7.49(0.97-57.63)$ & \\
\hline Other & 1.00 (reference) & \\
\hline Age (years) & $0.96(0.93-0.99)$ & 0.02 \\
\hline
\end{tabular}

$\mathrm{CI}=$ confidence interval; $\mathrm{NE}=$ not estimable

a Anterior resection with primary anastomosis and colostomy pooled

b Left-sided hemicolectomy and miscellaneous pooled

adjusted for length of follow-up using a univariate Cox regression. Age, level of anastomosis, and the persistence of postoperative symptoms appeared to be significant risk factors for recurrent divertitculitis. Younger age and persistent postoperative symptoms were significantly related to an increased risk for recurrence. This also held for either colorectal or colosigmoidal anastomosis compared with colostomy. A multivariate Cox regression analysis showed that these were independent risk factors for recurrent diverticulitis.

Gender, elective or emergency surgery, type of operation, and number of episodes were not significantly associated with a higher risk of recurrence.

\section{Discussion}

The primary aim of this large and well-documented study was to calculate the cumulative incidence of recurrent diverticulitis in patients who underwent emergency or elective 
surgery for diverticulitis and to identify possible risk factors associated with recurrence in a large group of patients with histology-confirmed diverticulitis. Diverticulitis recurred in about $9 \%$ of the cases and in two thirds within 5 years after initial surgery. Young patients and those with abdominal complaints were significantly at risk for recurrent diverticulitis.

Data from the 1960s and 1980s showed rates of clinically suspected recurrent diverticulitis after resection varying between 7 and $11 \%$ [10, 11, 14]. Slightly lower recurrence rates between 5 and $8 \%$ were found more recently, explained by the fact that recurrences had to be consistent with barium enema or CT findings [12, 13]. Our overall recurrence rate agrees with that of others, but the advantage of our long-term data lies in the estimated risk of recurrence adjusted for length of follow-up. The estimated risk of recurrence is $16 \%$ over 15 years, meaning that one of every six patients risks a recurrence after resection.

Younger age was a risk factor for recurrence independent of the greater lifetime exposure to diverticulosis in the present study. The pathogenic mechanism in young patients with diverticular disease presumably differs from that in older patients, in whom age-related weakening of the colonic wall seems to play an important role. Recent findings of histologic similarity between the colonic wall surrounding diverticula and biopsies of patients with inflammatory bowel disease are interesting in that they give a deeper understanding of the potential pathogenic mechanisms of diverticula formation and diverticulitis in young patients [15-17].

Abdominal symptoms persist after resection in up to $33 \%$ of the cases and are attributed by most authors to coexisting irritable bowel syndrome (IBS) based on considerable overlap between symptoms of both diseases [3, 18]. It cannot be ruled out that IBS accounted for a certain failure rate after surgery; however, this would be expected in patient groups lacking inflammatory changes in the resected specimens. Over $90 \%$ of resected bowel parts had histologic signs of inflammation in our series, making IBS an unlikely cause for persistent complaints, which is further supported by the finding that postoperative abdominal complaints are an independent risk factor for recurrent diverticulitis. This implies that persistence of symptoms after resection for complicated diverticulitis should be taken seriously and be properly investigated by physicians.

It has been found that recurrence rates are lower if the total sigmoid had been removed and a rectal anastomosis had been made $[11,12]$. We could not confirm this finding in the present study, wherein data on the level of anastomosis and type of operation were carefully extracted from the operative reports. Mean specimen length of $19.6 \mathrm{~cm}$ and $17.1 \mathrm{~cm}$, respectively, did not significantly differ between the recurrence and nonrecurrence groups, further suggesting that the extent of resection and type of anastomosis are not important factors for recurrence.

Elective surgical resection is advised after two episodes of uncomplicated diverticulitis, although recent reports suggest a more conservative and individualized approach [19]. The rationale for surgery is to prevent recurrent complicated diverticulitis and to reduce emergency procedures. We challenge this advise based on the findings that one of every six operated patient is at risk of recurrence, $22 \%$ of patients have persistent abdominal complaints, and $80 \%$ of patients needing emergency surgery for acute diverticulitis had no manifestation of diverticular disease prior to surgery. Moreover, recurrent diverticulitis was not associated with a higher percentage of emergency procedures. Long-term morbidity related to colostomy reversal further emphasizes that patient selection for elective surgery should be done with caution.

\section{Conclusion}

After surgical therapy for diverticulitis the estimated risk of recurrence is high and abdominal complaints are frequent. Younger age and the persistence of postoperative symptoms predict a recurrence of diverticulitis after resection. The clinical implication of these findings needs further investigation. Results of this study support the practice of careful selection of patients who will undergo surgery for diverticulitis.

Open Access This article is distributed under the terms of the Creative Commons Attribution Noncommercial License which permits any noncommercial use, distribution, and reproduction in any medium, provided the original author(s) and source are credited.

\section{References}

1. Painter NS, Burkitt DP (1971) Diverticular disease of the colon: a deficiency disease of Western civilization. Br Med J 2:450-454

2. Hughes LE (1969) Postmortem survey of diverticular disease of the colon. II. The muscular abnormality of the sigmoid colon. Gut 10:344-351

3. Parks TG (1975) Natural history of diverticular disease of the colon. Clin Gastroenterol 4:53-69

4. Roberts P, Abel M, Rosen L et al (1995) Practice parameters for sigmoid diverticulitis. The Standards Task Force American Society of Colon and Rectal Surgeons. Dis Colon Rectum 38:125-132

5. Chautems R, Ambrosetti P, Ludwig A et al (2001) Long-term follow-up after first acute episode of sigmoid diverticulitis: is surgery mandatory? Dis Colon Rectum 44:A5-A26

6. Maggard MA, Chandler CF, Schmit PJ (2001) Surgical diverticulitis: treatment options. Am Surg 67:1185-1189

7. Hinchey EJ, Schaal PG, Richards GK (1978) Treatment of perforated diverticular disease of the colon. Adv Surg 12:85-109 
8. Sarin S, Boulos PB (1994) Long-term outcome of patients presenting with acute complications of diverticular disease. Ann R Coll Surg Engl 76:117-120

9. Lorimer JW (1997) Is prophylactic resection valid as an indication for elective surgery in diverticular disease? Can J Surg 40:445-448

10. Leigh JE, Judd ES, Waugh JM (1962) Diverticulitis of the colon. Recurrence after apparently adequate segmental resection. Am J Surg 103:51-54

11. Benn PL, Wolff BG, Ilstrup DM (1986) Level of anastomosis and recurrent colonic diverticulitis. Am J Surg 151:269-271

12. Thaler K, Baig MK, Berho M (2003) Determinants of recurrence after sigmoid resection for uncomplicated diverticulitis. Dis Colon Rectum 46:385-388

13. Thorn M, Graf W, Stefansson T et al (2002) Clinical and functional results after elective colonic resection in 75 consecutive patients with diverticular disease. Am J Surg 183:7-11
14. Wolff BG, Ready RL, MacCarty RL et al (1984) Influence of sigmoid resection on progression of diverticular disease of the colon. Dis Colon Rectum 27:645-647

15. Koutroubakis IE, Antoniou P, Tzardi M et al (2005) The spectrum of segmental colitis associated with diverticulosis. Int $\mathrm{J}$ Colorectal Dis 20:28-32

16. Jani N, Finkelstein S, Blumberg D et al (2002) Segmental colitis associated with diverticulosis. Dig Dis Sci 47:1175-1181

17. Peppercorn MA (2004) The overlap of inflammatory bowel disease and diverticular disease. J Clin Gastroenterol 38:S8-S10

18. Munson KD, Hensien MA, Jacob LN et al (1996) Diverticulitis. A comprehensive follow-up. Dis Colon Rectum 39:318-322

19. Wong WD, Wexner SD, Lowry A et al (2000) Practice parameters for the treatment of sigmoid diverticulitis-supporting documentation. The Standards Task Force. The American Society of Colon and Rectal Surgeons. Dis Colon Rectum 43:290-297 